\title{
Compromise Ratio Method of MADM Problem Based on a New Interval-valued Intuitionistic Fuzzy Entropy
}

\author{
Haiping Ren ${ }^{1}$ and Wanzhen Liu $^{2}$ \\ 1. School of Software, Jiangxi University of Science and Technology, Nanchang \\ 330013, China \\ 2. Changsha Vocational \& Technical College, Changsha, 410010 China \\ ${ }^{1}$ chinarhp@163.com, ${ }^{2}$ liuwanzhenmath@163.com
}

\begin{abstract}
This article points out the shortcomings of the existing entropy measures, and puts forward a new improved interval-valued intuitionistic fuzzy (IVIF) entropy measure. The new entropy measure not only considers the absolute value of the deviation between membership and non-membership degrees, but also considers the effect of hesitancy degree of IVIF sets. For the two cases with attribute weights information unknown and partially known, new weighting methods are put forward by using the extended entropy method and by establishing the minimum entropy optimization model to solve the optimal weights. Further a compromise ratio method of intuitionistic fuzzy multi-attribute decision making (MADM) problems is put forward, and application examples proved the effectiveness and feasibility of the proposed methods.
\end{abstract}

Keywords: Interval-valued intuitionistic fuzzy set; fuzzy entropy; multi-attribute decision making; compromise ratio method

\section{Introduction}

With the complexity of the social economic environment and the incomplete understanding of human beings, there is a lot of ambiguity in real problems of management decision-making. In order to deal with the fuzzy phenomenon effectively, Zadeh [1] firstly proposed the concept of fuzzy set in 1965, and defined the corresponding algorithm. Since then, fuzzy sets have been widely applied in many fields such as automatic control, pattern recognition, medical diagnosis, management decision-making and so on. However, Zadeh's fuzzy sets only containing one membership which can only express two aspects of information, but it cannot be expressed the neutral state of neither support nor opposition. There are often many subjective and objective factors, such as the limited time, energy and the complete cognition of the objective things, which influence the decision maker's judgement. In many situations, there often exist different hesitancy degree or show a certain degree of lack of knowledge. To this end, professor Atanassov defined the concept of intuitionistic fuzzy (IF) sets [1], by adding a non-membership degree, it can describe the three kinds of state of support, opposition and neutrality, can more comprehensive and delicate to describe the fuzzy nature of objective things. IF sets can well describe the decision makers to the hesitation and uncertainty of judgment through the addition of a non-membership parameters. And they can describe the fuzzy characters of things comprehensively, and thus are a powerful and effective tool in expressing uncertain or fuzzy information in actual applications.

Due to the complexity and uncertainty of objective things and the limitation of the knowledge of decision maker, the degree of membership and non-membership is sometimes very difficult to express with crisp numbers, and the interval numbers can be very good. For this reason, Atanassov and Gargov expanded the intuitionistic fuzzy sets, 
and propose the concept of IVIF sets [2]. By interval numbers depicting the membership and non-membership degree, IVIF sets are more attractive than IF sets and can easily be quantified and execrated by decision-makers. IVIF sets are already widely applied in management decision problems [3-5].

In information theory, entropy is a measure of the average uncertainty. Zadeh [6] first introduced the concept of entropy into the fuzzy events in 1968, and then developed and gave the definition of fuzzy entropy. Luca De and Termini [7] was firstly constructed the concept of fuzzy entropy. Fuzzy entropy is an important information measure, which is applied in many fields such as MADM, image segmentation and pattern recognition [810]. Intuitionistic fuzzy sets have to be considered in two aspects [11]: the degree of uncertainty and the unknown degree, and the degree of uncertainty is represented by the degree of hesitation. Since the IVIF sets are a natural generalization of IF sets, the construction study of interval valued intuitionistic fuzzy entropy needs to consider the uncertainty and the unknown degree.

By analyzing the existing interval valued intuitionistic fuzzy entropy measure, we find that some scholars have proposed the influence of the fuzzy entropy formula of the intuitionistic fuzzy entropy on the interval valued intuitionistic fuzzy uncertainty. In this section, we will develop a new IVIF entropy measure which not only considers the absolute value of the deviation between membership and non-membership degrees, but also considers the effect of hesitancy degree of IVIF sets. For the two cases with attribute weights information unknown and partially known, new weighting methods are put forward by using the extended entropy method and by establishing the minimum entropy optimization model to solve the optimal weights. Further a compromise ratio method of intuitionistic fuzzy MADM problems is developed.

The organization of this paper is as follows: In Section 2, we recall some concepts and notations of IVIF sets. Section 3 firstly analyses the shortcoming of existing entropy measures and then puts forward a new IVIF entropy measure. Section 4 develops a new MADM methods based on the proposed IVIF entropy and the concept of compromise ratio method. Section 5 illustrates the effectiveness and feasibility of the proposed method. Finally, the conclusions are provided in Section 6.

\section{Preliminary Knowledge}

In what follows, some basic concepts of IF sets and IVIF sets are introduced to facilitate the discussions. Atanassov proposed the concept of IF sets in 1986, which is defined as in Definition 1.

Definition 1 [2] Let $X=\left\{x_{1}, x_{2}, \cdots, x_{n}\right\}$ be a finite universe of discourse, then

$$
U=\left\{<x_{j}, \mu_{U}\left(x_{j}\right), v_{U}\left(x_{j}\right)>\mid x_{j} \in X\right\}
$$

is called an IF set, which assigns to each element $x_{j}$ a membership degree $\mu_{U}\left(x_{j}\right)$ and a nonmembership degree $v_{U}\left(x_{j}\right)$.

Here $\mu_{U}\left(x_{j}\right) \in[0,1], v_{U}\left(x_{j}\right) \in[0,1]$ and $\pi_{U}\left(x_{j}\right)=1-\mu_{U}\left(x_{j}\right)-v_{U}\left(x_{j}\right)$ is called the hesitation degree or intuitionistic index of an element $x_{j}$ to $U$. It is the degree of indeterminacy membership of the element $x_{j}$ to the IF set $U$. It is a good tool to reflect the uncertain information, thus it can also help the decision maker to descript the fuzzy information. Obviously, $0 \leq \pi_{U}\left(x_{j}\right) \leq 1$ for every $x_{j} \in X$. And if $\pi_{U}\left(x_{j}\right)=0$, then the IF set $U$ is reduced to a fuzzy set, i.e., $U=\left\{<\mu_{U}\left(x_{j}\right), 1-\mu_{U}\left(x_{j}\right)>\mid x_{j} \in X\right\}$.

In some situation, it is very difficult to use crisp numbers to express $\mu_{U}\left(x_{j}\right)$ and $v_{U}\left(x_{j}\right)$ precisely for the complexity and uncertainties of the objective things. But we can use 
intervals to express them. So, Atanassov and Gargov extended the IF set to the IVIF set in 1989, and gave the definition of the IVIFS as in Definition 2.

Definition 2 [2] Let $X=\left\{x_{1}, x_{2}, \cdots, x_{n}\right\}$ be a finite universe of discourse, then

$$
\tilde{U}=\left\{<x_{j}, \tilde{\mu}_{\tilde{U}}\left(x_{j}\right), \tilde{v}_{\tilde{U}}\left(x_{j}\right)>\mid x_{j} \in X\right\}
$$

is called an IVIF set, where $\tilde{\mu}_{\tilde{U}}\left(x_{j}\right)$ and $\tilde{v}_{\tilde{U}}\left(x_{j}\right)$ are intervals, where $\tilde{\mu}_{\tilde{U}}\left(x_{j}\right)=\left[\mu_{\tilde{U}}^{-}\left(x_{j}\right), \mu_{\tilde{U}}^{+}\left(x_{j}\right)\right] \quad$ and $\quad \tilde{v}_{\tilde{U}}\left(x_{j}\right)=\left[v_{\tilde{U}}^{-}\left(x_{j}\right), v_{\tilde{U}}^{+}\left(x_{j}\right)\right] \quad$. The element $<x_{j}, \tilde{\mu}_{\tilde{U}}\left(x_{j}\right), \tilde{v}_{\tilde{U}}\left(x_{j}\right)>$ is called an IVIF number [12]. The hesitation degree of an IVIF number $\left(\tilde{\mu}_{\tilde{U}}\left(x_{j}\right), \tilde{v}_{\tilde{U}}\left(x_{j}\right)\right)$ can be defined as $\tilde{\pi}_{\tilde{U}}\left(x_{j}\right)=\left[\pi_{\tilde{U}}^{-}\left(x_{j}\right), \pi_{\tilde{U}}^{+}\left(x_{j}\right)\right]$, where $\pi_{\tilde{U}}^{-}\left(x_{j}\right)=1-\mu_{\tilde{U}}^{+}\left(x_{j}\right)-v_{\tilde{U}}^{+}\left(x_{j}\right)$ and $\pi_{\tilde{U}}^{+}\left(x_{j}\right)=1-\mu_{\tilde{U}}^{-}\left(x_{j}\right)-v_{\tilde{U}}^{-}\left(x_{j}\right)$, for all $x_{j} \in X$.

We briefly denote an IVIF number $<\tilde{\mu}_{\tilde{U}}\left(x_{j}\right), \tilde{v}_{\tilde{U}}\left(x_{j}\right)>$ by $\tilde{A}=<\tilde{\mu}_{\tilde{A}}, \tilde{\mathcal{U}}_{\tilde{A}}>$ or $\tilde{A}=<\tilde{\mu}_{\tilde{A}}, \tilde{v}_{\tilde{A}}, \tilde{\pi}_{\tilde{A}}>$, where

$$
\begin{gathered}
\tilde{\mu}_{\tilde{A}}=\left[\mu_{\tilde{A}}^{-}, \mu_{\tilde{A}}^{+}\right] \subset[0,1], \tilde{v}_{\tilde{A}}=\left[v_{\tilde{A}}^{-}, v_{\tilde{A}}^{+}\right] \subset[0,1], \mu_{\tilde{A}}^{+}+\tilde{v}_{\tilde{A}}^{+} \leq 1 \\
\tilde{\pi}_{\tilde{A}}=\left[\pi_{\tilde{A}}^{-}, \pi_{\tilde{A}}^{+}\right] \subset[0,1], \pi_{\tilde{A}}^{-}=1-\mu_{\tilde{A}}^{+}-\tilde{v}_{\tilde{A}}^{+}, \pi_{\tilde{A}}^{+}=1-\mu_{\tilde{A}}^{-}-\tilde{v}_{\tilde{A}}^{-}
\end{gathered}
$$

Definition 3 [2] Let $\tilde{A}_{i}=<\tilde{\mu}_{\bar{A}_{i}}, \tilde{\mathcal{A}}_{\bar{A}_{i}}>(i=1,2)$ be two any IVIF number, then

(1) If $\mu_{\bar{A}_{1}}^{-} \leq \mu_{\bar{A}_{2}}^{-}, \mu_{\bar{A}_{1}}^{+} \leq \mu_{\bar{A}_{2}}^{+}$and $v_{\bar{A}_{1}}^{-} \geq v_{\bar{A}_{2}}^{-}, v_{\bar{A}_{1}}^{+} \geq v_{\bar{A}_{2}}^{+}$, then $\tilde{A}_{1}$ is no larger than $\tilde{A}_{2}$, and noted by $\tilde{A}_{1} \leq \tilde{A}_{2}$;

(2) If $\tilde{A}_{1} \leq \tilde{A}_{2}$ and $\tilde{A}_{1} \geq \tilde{A}_{2}$, then $\tilde{A}_{1}$ is equal to $\tilde{A}_{2}$, and noted by $\tilde{A}_{1}=\tilde{A}_{2}$.

By Definition 3, $\tilde{A}^{*}=<[1,1],[0,0]>$ is the largest IVIF number; $\tilde{A}^{-}=<[0,0],[1,1]>$ is the smallest IVIF number.

Definition 4. Let $\tilde{A}=\left\{<x_{i}, \tilde{\mu}_{\bar{A}}\left(x_{i}\right), \tilde{v}_{\bar{A}}\left(x_{i}\right)>\mid x_{i} \in X\right\}$ and $\tilde{B}=\left\{<x_{i}, \tilde{\mu}_{\tilde{B}}\left(x_{i}\right), \tilde{v}_{\tilde{B}}\left(x_{i}\right)>\mid x_{i} \in X\right\}$ be two IVIF sets, then the following operations can be founded in [2]:

(3) The complementary set of $\tilde{A}$ denoted by $\tilde{A}^{C}$, is $\tilde{A}^{C}=\left\{<x_{i}, \tilde{v}_{\tilde{A}}\left(x_{i}\right), \tilde{\mu}_{\tilde{A}}\left(x_{i}\right)>\mid x_{i} \in X\right\}$;

(4) $\tilde{A} \preceq \tilde{B}$ called $\tilde{A}$ less fuzzy than $\tilde{B}$, i.e., for $\forall x_{i} \in X$,

If $\tilde{\mu}_{\tilde{B}}\left(x_{i}\right) \leq \tilde{v}_{\tilde{B}}\left(x_{i}\right)$, then $\tilde{\mu}_{\tilde{A}}\left(x_{i}\right) \leq \tilde{\mu}_{\tilde{B}}\left(x_{i}\right), \tilde{v}_{\tilde{A}}\left(x_{i}\right) \geq \tilde{v}_{\tilde{B}}\left(x_{i}\right)$;

If $\tilde{\mu}_{\tilde{B}}\left(x_{i}\right) \geq \tilde{v}_{\tilde{B}}\left(x_{i}\right)$, then $\tilde{\mu}_{\tilde{A}}\left(x_{i}\right) \geq \tilde{\mu}_{\tilde{B}}\left(x_{i}\right), \tilde{v}_{\tilde{A}}\left(x_{i}\right) \leq \tilde{v}_{\tilde{B}}\left(x_{i}\right)$.

Definition 5. Let $\tilde{A}=\left\{<x_{i}, \tilde{\mu}_{\bar{A}}\left(x_{i}\right), \tilde{v}_{\bar{A}}\left(x_{i}\right)>\mid x_{i} \in X\right\}$ and $\tilde{B}=\left\{<x_{i}, \tilde{\mu}_{\tilde{B}}\left(x_{i}\right), \tilde{v}_{\tilde{B}}\left(x_{i}\right)>\mid x_{i} \in X\right\}$ be two IVIF sets and the weight of $x_{i}$ is $w_{i}$. Then the weighted Hamming distance of $\tilde{A}$ and $\tilde{B}$ is defined as[12]:

$$
\begin{aligned}
& d(\tilde{A}, \tilde{B})=\frac{1}{4 n} \sum_{j=1}^{n} w_{j}[ {\left[\left|\mu_{A}^{-}\left(x_{j}\right)-\mu_{B}^{-}\left(x_{j}\right)\right|+\left|\mu_{A}^{+}\left(x_{j}\right)-\mu_{B}^{+}\left(x_{j}\right)\right|+\left|v_{A}^{-}\left(x_{j}\right)-v_{B}^{-}\left(x_{j}\right)\right|\right.} \\
&\left.+\left|v_{A}^{+}\left(x_{j}\right)-v_{B}^{+}\left(x_{j}\right)\right|+\left|\pi_{A}^{-}\left(x_{j}\right)-\pi_{B}^{-}\left(x_{j}\right)\right|+\left|\pi_{A}^{+}\left(x_{j}\right)-\pi_{B}^{+}\left(x_{j}\right)\right|\right]
\end{aligned}
$$

In the following discussion, we always suppose that $\operatorname{IVIFS} s(Z)$ is the set of all IVIF sets defined in $X$. 
Definition 6 Let $\tilde{A}=\left\{<x_{i}, \tilde{\mu}_{\bar{A}}\left(x_{i}\right), \tilde{v}_{\bar{A}}\left(x_{i}\right)>\mid x_{i} \in X\right\}$ and $\tilde{B}=\left\{<x_{i}, \tilde{\mu}_{\tilde{B}}\left(x_{i}\right), \tilde{v}_{\tilde{B}}\left(x_{i}\right)>\mid x_{i} \in X\right\}$ be two IVIF sets . A map $E: \operatorname{IVIFS} s(Z) \rightarrow[0,1]$ is called the IVIF entropy, if it satisfies the following properties [13]:

(i) $E(\tilde{A})=0$ if and only if $\tilde{A}$ is a crisp set;

(ii) $E(\tilde{A})=1$ if and only if $\tilde{\mu}_{\tilde{A}}\left(x_{i}\right)=\tilde{v}_{\tilde{A}}\left(x_{i}\right), \forall x_{i} \in X$;

(iii) $E(\tilde{A})=E\left(\tilde{A}^{C}\right)$;

(iv) If $\tilde{A} \preceq \tilde{B}$, then $E(\tilde{A}) \leq E(\tilde{B})$.

\section{A New Effective IVIF Entropy}

First we review several already existing IVIF entropy measures in reference. By analyzing the existing IVIF entropy measure, we find that some existing IVIF entropy measures without considering with the influence of the hestiation, such as Vlachos' IVIF entropy [14]:

$$
E_{1}(A)=1-\sqrt{\frac{1}{2 n} \sum_{i=1}^{n}\left(\left|\mu_{A}^{-}\left(x_{i}\right)-v_{A}^{-}\left(x_{i}\right)\right|^{2}+\left|\mu_{A}^{+}\left(x_{i}\right)-v_{A}^{+}\left(x_{i}\right)\right|^{2}\right)}
$$

and Ye's IVIF entropy [15]:

$$
\begin{aligned}
E_{2}(\tilde{A})= & \left\{\sin \frac{\pi \times\left[1+\mu_{\tilde{A}}^{-}\left(x_{i}\right)+p \Delta \mu_{\tilde{A}}\left(x_{i}\right)-v_{\tilde{A}}^{-}\left(x_{i}\right)-p \Delta v_{\tilde{A}}\left(x_{i}\right)\right]}{4}\right. \\
& \left.+\sin \frac{\pi \times\left[1-\mu_{\tilde{A}}^{-}\left(x_{i}\right)-p \Delta \mu_{\tilde{A}}\left(x_{i}\right)+v_{\tilde{A}}^{-}\left(x_{i}\right)+p \Delta v_{\tilde{A}}\left(x_{i}\right)\right]}{4}-1\right\} \times \frac{1}{\sqrt{2}-1}
\end{aligned}
$$

where $\Delta \mu_{\tilde{A}}\left(x_{i}\right)=\mu_{\tilde{A}}^{+}\left(x_{i}\right)-\mu_{\tilde{A}}^{-}\left(x_{i}\right), \Delta v_{\tilde{A}}\left(x_{i}\right)=v_{\tilde{A}}^{+}\left(x_{i}\right)-v_{\tilde{A}}^{-}\left(x_{i}\right)$.

Example 1. Let $\tilde{A}=\langle[0.4,0.5],[0.3,0.4]\rangle, \tilde{B}=\langle[0.2,0.3],[0.1,0.2]\rangle, \tilde{C}=\langle[0.6,0.6]$, $[0.2,0.2]\rangle$ and $\tilde{D}=\langle[0.7,0.7],[0.3,0.3]\rangle$ be four IVIF numbers. Intuitively, we can see that $\tilde{B}$ is more fuzzy than $\tilde{A}$, and $\tilde{C}$ is more fuzzy than $\tilde{D}$. But $E_{1}(\tilde{A})=E_{1}(\tilde{B})=0.9000$ and $E_{2}(\tilde{C})=E_{2}(\tilde{D})=0.8329$, which are not consistent with our intuition.

Another scholars considered the influence of hesitation degree in the construction ofIVIF entropy, such as Wei's IVIF entropy [13]:

$$
E_{3}(\tilde{A})=\frac{1}{n} \sum_{i=1}^{n} \frac{2-\left|\mu_{A}^{-}\left(x_{i}\right)-v_{A}^{-}\left(x_{i}\right)\right|-\left|\mu_{A}^{+}\left(x_{i}\right)-v_{A}^{+}\left(x_{i}\right)\right|+\pi_{A}^{-}\left(x_{i}\right)+\pi_{A}^{+}\left(x_{i}\right)}{\left.2+\mid x_{i}\right)-v_{A}^{-}\left(x_{i}\right)|+| \mu_{A}^{+}\left(x_{i}\right)-v_{A}^{+}\left(x_{i}\right) \mid+\pi_{A}^{-}\left(x_{i}\right)+\pi_{A}^{+}\left(x_{i}\right)}
$$

and Jin et al's IVIF entropy [16] :

$$
E_{4}(\tilde{A})=\frac{1}{n} \sum_{i=1}^{n} \frac{\min \left\{F_{Q}\left(\tilde{\mu}_{\tilde{A}}\left(x_{i}\right)\right), F_{Q}\left(\tilde{v}_{\tilde{A}}\left(x_{i}\right)\right)\right\}+\pi_{F_{Q}\left(x_{i}\right)}}{\max \left\{F_{Q}\left(\tilde{\mu}_{\tilde{A}}\left(x_{i}\right)\right), F_{Q}\left(\tilde{v}_{\tilde{A}}\left(x_{i}\right)\right)\right\}+\pi_{F_{Q}\left(x_{i}\right)}}
$$

where $\left.\pi_{F_{Q}\left(x_{i}\right)}=1-F_{Q}\left(\tilde{\mu}_{\tilde{A}}\left(x_{i}\right)\right)-F_{Q}\left(\tilde{v}_{\tilde{A}}\left(x_{i}\right)\right)\right\}, \quad F_{Q}(\tilde{a})$ is the Yager's COWA operator of interval number $\tilde{a}=\left[a^{-}, a^{+}\right]$, which has the following form:

$$
F_{Q}(\tilde{a})=\int_{0}^{1} \frac{d Q(y)}{d y}\left[a^{+}-y\left(a^{+}-a^{-}\right)\right] d y .
$$

If $\lambda=\int_{o}^{1} Q(y) d y$, then $F_{Q}\left(\left[a^{-}, a^{+}\right]\right)=\lambda a^{+}+(1-\lambda) a^{-}$. 
Example 2. Let us consider two candidates $\tilde{A}$ and $\tilde{B}$, the support ratio of $\tilde{A}=<[0.2,0.2],[0.067,0.067]>$ is $40 \%$, while the support ratio $\tilde{B}=<[0.4,0.4],[0.2,0.2]>$ is $60 \%$, then we will select $\tilde{B}$ is as the better candidate than $\tilde{A}$. This voting result is expressed that $\tilde{A}$ is more fuzzy than B. We note that, if $\lambda=0.5$, then $E_{4}(\tilde{A})=E_{3}(\tilde{A})$. In this case, we can get the result $E_{4}(\tilde{A})=E_{4}(\tilde{B})$, which is not consistent with the true situation.

The main task of this section is to put forward an effective IVIF entropy, which not only considers the absolute value of the deviation between membership and nonmembership degrees, but also considers the effect of hesitancy degree of IVIF sets.

Let $\left.A=\left\{<x_{i},\left[\mu_{A L}\left(x_{i}\right), \mu_{A U}\left(x_{i}\right)\right],\left[v_{A L}\left(x_{i}\right), v_{A U}\left(x_{i}\right)\right]\right\rangle \mid x_{i} \in X, i=1,2, \ldots, n\right\}$ be an IVIF set, we define a new information measure of $A$ as follows:

$$
E(A)=\frac{1}{n} \sum_{i=1}^{n} \cos \frac{\left|\mu_{A L}^{2}\left(x_{i}\right)-v_{A L}^{2}\left(x_{i}\right)\right|+\left|\mu_{A U}^{2}\left(x_{i}\right)-v_{A U}^{2}\left(x_{i}\right)\right|}{4} \pi
$$

Equation (8) can also be rewritten as

$$
E(A)=\frac{1}{n} \sum_{i=1}^{n} \cos \frac{\left|\left(\mu_{A L}\left(x_{i}\right)-v_{A L}\left(x_{i}\right)\right)\left(1-\pi_{A U}\left(x_{i}\right)\right)\right|+\left|\left(\mu_{A U}\left(x_{i}\right)-v_{A U}\left(x_{i}\right)\right)\left(1-\pi_{A L}\left(x_{i}\right)\right)\right|}{4} \pi
$$

From Equation (9), we can see that $E(A)$ not only considers the deviation between membership with nonmembership degrees( i.e. $\left.\mu_{A L}\left(x_{i}\right)-v_{A L}\left(x_{i}\right), \mu_{A U}\left(x_{i}\right)-v_{A U}\left(x_{i}\right)\right)$, but also considers the hesitancy degree of the IVIF set( i.e. $\left.\pi_{A L}\left(x_{i}\right), \pi_{A U}\left(x_{i}\right)\right)$.

Theorem 1. The measure given by Equation (9) is an IVIF entropy.

Proof. To prove the measure given by Equation (9) is an IVIF entropy, we only need to prove it satisfies the properties in definition 6. Obviously, for every $x_{i}$, we have: $0 \leq E(A) \leq 1$.

(i) Let $A$ be a crisp set, i.e., for $\forall x_{i} \in X$, we have $\tilde{\mu}_{A}\left(x_{i}\right)=[0,0], \tilde{v}_{A}\left(x_{i}\right)=[1,1]$ or $\tilde{\mu}_{A}\left(x_{i}\right)=[1,1], \tilde{v}_{A}\left(x_{i}\right)=[0,0]$. It is obvious that $E(A)=0$.

If $E(A)=0$, i.e., $E(A)=\frac{1}{n} \sum_{i=1}^{n} \cos \frac{\left|\mu_{A L}^{2}\left(x_{i}\right)-v_{A L}^{2}\left(x_{i}\right)\right|+\left|\mu_{A U}^{2}\left(x_{i}\right)-v_{A U}^{2}\left(x_{i}\right)\right|}{4} \pi=0$, then $\forall x_{i} \in X$, we have

$$
\cos \frac{\left|\mu_{A L}^{2}\left(x_{i}\right)-v_{A L}^{2}\left(x_{i}\right)\right|+\left|\mu_{A U}^{2}\left(x_{i}\right)-v_{A U}^{2}\left(x_{i}\right)\right|}{4} \pi=0,
$$

thus $\left|\mu_{A L}^{2}\left(x_{i}\right)-v_{A L}^{2}\left(x_{i}\right)\right|=1 \quad, \quad\left|\mu_{A U}^{2}\left(x_{i}\right)-v_{A U}^{2}\left(x_{i}\right)\right|=1 \quad, \quad$ then we have $\tilde{\mu}_{A}\left(x_{i}\right)=[0,0], \tilde{v}_{A}\left(x_{i}\right)=[1,1]$ or $\tilde{\mu}_{A}\left(x_{i}\right)=[1,1], \tilde{v}_{A}\left(x_{i}\right)=[0,0]$, Therefore $A$ is a crisp set.

(ii) Let $\tilde{\mu}_{\tilde{A}}\left(x_{i}\right)=\tilde{v}_{\tilde{A}}\left(x_{i}\right), \forall x_{i} \in X$, from Equation (9), we have $E(A)=1$.

Now we assume that $E(A)=1$, then for all $x_{i} \in X$, we have:

$$
\cos \frac{\left|\mu_{A L}^{2}\left(x_{i}\right)-v_{A L}^{2}\left(x_{i}\right)\right|+\left|\mu_{A U}^{2}\left(x_{i}\right)-v_{A U}^{2}\left(x_{i}\right)\right|}{4} \pi=1 \text {, }
$$

then $\frac{\left|\mu_{A L}^{2}\left(x_{i}\right)-v_{A L}^{2}\left(x_{i}\right)\right|+\left|\mu_{A U}^{2}\left(x_{i}\right)-v_{A U}^{2}\left(x_{i}\right)\right|}{4}=0$, we can obtain the conclusion $\mu_{A L}\left(x_{i}\right)=v_{A L}\left(x_{i}\right), \mu_{A U}\left(x_{i}\right)=v_{A U}\left(x_{i}\right)$ for all $x_{i} \in X$. 
(iii) By $\tilde{A}^{C}=\left\{<x_{i}, \tilde{v}_{\bar{A}}\left(x_{i}\right), \tilde{\mu}_{\bar{A}}\left(x_{i}\right)>\mid x_{i} \in X\right\}$ and Equation (9), we have:

$$
\begin{aligned}
E\left(\tilde{A}^{C}\right) & =\frac{1}{n} \sum_{i=1}^{n} \cos \frac{\left|v_{A L}^{2}\left(x_{i}\right)-\mu_{A L}^{2}\left(x_{i}\right)\right|+\left|v_{A U}^{2}\left(x_{i}\right)-\mu_{A U}^{2}\left(x_{i}\right)\right|}{4} \pi \\
& =\frac{1}{n} \sum_{i=1}^{n} \cos \frac{\left|\mu_{A L}^{2}\left(x_{i}\right)-v_{A L}^{2}\left(x_{i}\right)\right|+\left|\mu_{A U}^{2}\left(x_{i}\right)-v_{A U}^{2}\left(x_{i}\right)\right|}{4} \pi . \\
& =E(A)
\end{aligned}
$$

(iv) If $\tilde{A} \preceq \tilde{B}$, then for $\forall x_{i} \in X$,

For the one case $\tilde{\mu}_{\bar{B}}\left(x_{i}\right) \leq \tilde{v}_{\bar{B}}\left(x_{i}\right), \tilde{\mu}_{\tilde{A}}\left(x_{i}\right) \leq \tilde{\mu}_{\tilde{B}}\left(x_{i}\right), \tilde{v}_{\tilde{A}}\left(x_{i}\right) \geq \tilde{v}_{\tilde{B}}\left(x_{i}\right)$, that is

$$
\begin{gathered}
\mu_{B L}\left(x_{i}\right) \leq v_{B L}\left(x_{i}\right), \mu_{B U}\left(x_{i}\right) \leq v_{B U}\left(x_{i}\right), \mu_{A L}\left(x_{i}\right) \leq \mu_{B L}\left(x_{i}\right), \mu_{A U}\left(x_{i}\right) \leq \mu_{B U}\left(x_{i}\right), \\
\tilde{\mu}_{\tilde{A}}\left(x_{i}\right) \geq \tilde{\mu}_{\tilde{B}}\left(x_{i}\right), \tilde{v}_{\tilde{A}}\left(x_{i}\right) \leq \tilde{v}_{\tilde{B}}\left(x_{i}\right) .
\end{gathered}
$$

Then

Thus we have

$$
\begin{aligned}
& \mu_{A L}^{2}\left(x_{i}\right)-v_{A L}^{2}\left(x_{i}\right) \leq \mu_{B L}^{2}\left(x_{i}\right)-v_{B L}^{2}\left(x_{i}\right) \leq 0 \\
& \mu_{A U}^{2}\left(x_{i}\right)-v_{A U}^{2}\left(x_{i}\right) \leq \mu_{B U}^{2}\left(x_{i}\right)-v_{B U}^{2}\left(x_{i}\right) \leq 0
\end{aligned}
$$

and

$$
\begin{aligned}
& \left|\mu_{A L}^{2}\left(x_{i}\right)-v_{A L}^{2}\left(x_{i}\right)\right| \geq\left|\mu_{B L}^{2}\left(x_{i}\right)-v_{B L}^{2}\left(x_{i}\right)\right| \\
& \left|\mu_{A U}^{2}\left(x_{i}\right)-v_{A U}^{2}\left(x_{i}\right)\right| \geq\left|\mu_{B U}^{2}\left(x_{i}\right)-v_{B U}^{2}\left(x_{i}\right)\right|
\end{aligned}
$$

$$
\left|\mu_{A L}^{2}\left(x_{i}\right)-v_{A L}^{2}\left(x_{i}\right)\right|+\left|\mu_{A U}^{2}\left(x_{i}\right)-v_{A U}^{2}\left(x_{i}\right)\right| \geq\left|\mu_{B L}^{2}\left(x_{i}\right)-v_{B L}^{2}\left(x_{i}\right)\right|+\left|\mu_{B U}^{2}\left(x_{i}\right)-v_{B U}^{2}\left(x_{i}\right)\right|
$$

So, we can get

$$
\begin{aligned}
& \cos \frac{\left|\mu_{A L}^{2}\left(x_{i}\right)-v_{A L}^{2}\left(x_{i}\right)\right|+\left|\mu_{A U}^{2}\left(x_{i}\right)-v_{A U}^{2}\left(x_{i}\right)\right|}{4} \pi \\
\leq & \cos \frac{\left|\mu_{B L}^{2}\left(x_{i}\right)-v_{B L}^{2}\left(x_{i}\right)\right|+\left|\mu_{B U}^{2}\left(x_{i}\right)-v_{B U}^{2}\left(x_{i}\right)\right|}{4} \pi
\end{aligned}
$$

That is $E(A) \leq E(B)$.

Simarily, we can prove that for the case of $\tilde{\mu}_{\tilde{B}}\left(z_{i}\right) \geq \tilde{\mathcal{V}}_{\tilde{B}}\left(z_{i}\right), \tilde{\mu}_{\tilde{A}}\left(z_{i}\right) \geq \tilde{\mu}_{\tilde{B}}\left(z_{i}\right)$ and $\tilde{v}_{\tilde{A}}\left(z_{i}\right) \leq \tilde{v}_{\tilde{B}}\left(z_{i}\right)$, the result $E(\tilde{A}) \leq E(\tilde{B})$ can also be proved.

Then we get the conclusion: If $\tilde{A} \preceq \tilde{B}$, then $E(\tilde{A}) \leq E(\tilde{B})$.

\section{A New MADM Method based on Proposed IVIF Entropy}

Suppose that there exists an alternative set $A=\left\{A_{1}, A_{2}, \cdots, A_{m}\right\}$ consisting of $n$ noninferior alternatives from which the most preferred alternative is to be selected. A MADM method is to find the best alternative from a set of $m$ alternatives with respect to a set $O=\left\{o_{1}, o_{2}, \cdots, o_{n}\right\}$ of $n$ attributes. Suppose that the ratings of alternatives $A_{i}$ on attributes $o_{j}$ are expressed with the IVIF number $\tilde{a}_{i j}=<\left[\mu_{i j L}, \mu_{i j U}\right],\left[v_{i j L}, v_{i j U}\right]>$, where $\left[\mu_{i j L}, \mu_{i j U}\right]$ and $\left[v_{i j L}, v_{i j U}\right]$ are intervals, which express the membership (satisfactory) and nonmembership (nonsatisfactory) degree of the alternative $A_{i}$ on the attribute $o_{j}$ with respect to the fuzzy concept "excellence" given by the decision maker so that they satisfy the conditions: $\mu_{i j L}, \mu_{i j U}, v_{i j L}, v_{i j U} \in[0,1]$ and $0 \leq \mu_{i j U}+v_{i j U} \leq 1(i=1,2, \cdots, m ; j=1,2, \cdots, n)$.

Thus, a MADM problem can be expressed with the following decision matrix: 


$$
\left.D=\left(\tilde{a}_{i j}\right)_{m \times n}=A_{2} \quad \begin{array}{ccccc}
o_{1} & o_{2} & \cdots & o_{n} \\
\vdots & A_{11} & \tilde{a}_{12} & \cdots & \tilde{a}_{1 n} \\
A_{m} & \tilde{a}_{21} & \tilde{a}_{22} & \cdots & \tilde{a}_{2 n} \\
\vdots & \vdots & \ddots & \vdots \\
\tilde{a}_{m 1} & \tilde{a}_{m 2} & \cdots & \tilde{a}_{m n}
\end{array}\right)
$$

In real decision situations, evaluated attributes may have different importance for the decision process. Let $\boldsymbol{W}=\left(w_{1}, w_{2}, \ldots, w_{n}\right)^{T}$ be the weight vector of all attributes, where $0 \leq w_{j} \leq 1 \quad(j=1,2, \cdots, n)$ are weights of attributes $o_{j} \in O$, and satisfy $\sum_{j=1}^{n} w_{j}=1$. The information of attribute weight is usually completely unknown or partially known due to the insufficient knowledge or limitation of time of decision makers. Therefore, the determination of attribute weights is an important issue in MADM problems. Then in this section, we will develop two methods to determine the weights of attributes for the above-mentioned two cases, respectively.

\subsection{Weight Determining Method with Unknown Attribute Weights Information}

When the attribute weights are completely unknown, we can use the proposed IVIF entropy to determine the attribute weights based on the concept of entropy weighting method. The calculate method is given as follows:

$$
w_{j}=\frac{1-e_{j}}{n-\sum_{j=1}^{n} e_{j}}, \quad j=1,2, \ldots, n
$$

where $e_{j}=\frac{1}{m} \sum_{i=1}^{m} E\left(\tilde{a}_{i j}\right)(j=1,2, \cdots, n)$, and $E\left(\tilde{a}_{i j}\right)=\cos \frac{\left|\mu_{i j L}^{2}-v_{i j L}^{2}\right|+\left|\mu_{i j U}^{2}-v_{i j U}^{2}\right|}{4} \pi$ is the IVIF entropy of $\tilde{a}_{i j}=<\left[\mu_{i j L}, \mu_{i j U}\right],\left[v_{i j L}, v_{i j U}\right]>$.

\subsection{Weight Determining Method with Partial Attribute Weight Information}

Generally, there will have more constraint conditions for the weight vector $\boldsymbol{W}=\left(w_{1}, w_{2}, \ldots, w_{n}\right)^{T}$. We denote $\boldsymbol{H}$ as the set of the known weight information. To determine the attribute weights for MADM problem with attribute weights partially known under IVIF environment, Liu and Ren [17] determined the attribute weights by establishing a programming model according to the minimum entropy principle. In this paper, we will use the new IVIF entropy measure to determine the attribute weights and the method is similarly with Liu and Ren [17]. The specific process is given as follows.

To rank the alternatives according to the decision matrix $D=\left(\tilde{a}_{i j}\right)_{m \times n}$, we propose a method to obtain the attribute weight vector by means of the proposed IVIF entropy measure. Entropy measure describes the degree of the fuzziness and intuitionism. The smaller the intuitionistic fuzzy entropy, the smaller of the fuzzy degree of attribute evaluation information, thus the more decision-making certainty information will be. Hence, we can utilize the principle of minimum entropy value to get the weight vector of attribute by computing the following programming: 


$$
\begin{aligned}
& \min E\left(A_{i}\right)=\sum_{j=1}^{n} w_{j} E\left(\tilde{a}_{i j}\right)=\sum_{j=1}^{n} w_{j} \cos \frac{\left|\mu_{i j L}^{2}-v_{i j L}^{2}\right|+\left|\mu_{i j U}^{2}-v_{i j U}^{2}\right|}{4} \pi \\
& \text { s.t. } \quad \sum_{j=1}^{n} w_{j}=1 \\
& \quad \boldsymbol{W} \in H
\end{aligned}
$$

Because each alternative is fair competition, the weight coefficient with respect to the same attribute should be also equal, thus we get the following optimization model:

$$
\begin{aligned}
& \min E=\sum_{i=1}^{m} E\left(A_{i}\right)=\sum_{i=1}^{m} \sum_{j=1}^{n} w_{j} \cos \frac{\left(\mu_{i j L}^{2}-v_{i j L}^{2}\right)^{2}+\left(\mu_{i j U}^{2}-v_{i j U}^{2}\right)^{2}}{4} \pi \\
& \text { s.t. } \quad \sum_{j=1}^{n} w_{j}=1 \\
& \quad \boldsymbol{W} \in H
\end{aligned}
$$

Hence, by solving the Equation (14), the optimal solution $\boldsymbol{W}^{*}=\arg \min E$ is chosen as the optimal attribute weights.

\subsection{The New MADM method based on the Proposed IVIF Entropy}

In this subsection, we put forward the new MADM method based on the abovementioned work and the concept of compromise ratio method. The specific calculation steps are given as follows:

Step 1. Calculate the attribute weights according to Section 4.1 and Section 4.2;

Step 2. Determine the positive ideal solution (PIS) and negative ideal solution (NIS) of the intuitionistic fuzzy MADM problem.

The PIS is defined as: $A^{*}=\left(\tilde{a}_{1}^{*}, \tilde{a}_{2}^{*}, \cdots, \tilde{a}_{n}^{*}\right)$, where $\tilde{a}_{j}^{*}=<[1,1],[0,0]>(j=1,2, \cdots, n)$.

The NIS is defined as: $A^{-}=\left(\tilde{a}_{1}^{-}, \tilde{a}_{2}^{-}, \cdots, \tilde{a}_{n}^{-}\right)$, where $\tilde{a}_{j}^{-}=<[0,0],[1,1]>(j=1,2, \cdots, n)$.

Step 3. According to the weighted Hamming distance measure in Definition 3, the distance measures between alternative $A_{i}$ with PIS and NIS are calculated respectively as follows:

$$
\begin{aligned}
d\left(A_{i}, A^{*}\right) & =\frac{1}{4} \sum_{j=1}^{n} w_{j}\left(\left|1-\mu_{i j L}\right|+\left|1-\mu_{i j U}\right|+\left|v_{i j L}-0\right|+\left|v_{i j U}-0\right|+\left|\pi_{i j L}-0\right|+\left|\pi_{i j U}-0\right|\right) \\
& =\frac{1}{4} \sum_{j=1}^{n} w_{j}\left(\left|1-\mu_{i j L}\right|+\left|1-\mu_{i j U}\right|+\left|v_{i j L}\right|+\left|v_{i j U}\right|+\left|1-\mu_{i j U}-v_{i j U}\right|+\left|1-\mu_{i j L}-v_{i j L}\right|\right) \\
& =\frac{1}{2} \sum_{j=1}^{n} w_{j}\left(2-\mu_{i j L}-\mu_{i j U}\right) \\
d\left(A_{i}, A^{-}\right) & =\frac{1}{4} \sum_{j=1}^{n} w_{j}\left(\left|\mu_{i j L}\right|+\left|\mu_{i j U}\right|+\left|1-v_{i j L}\right|+\left|1-v_{i j U}\right|+\left|\pi_{i j L}-0\right|+\left|\pi_{i j U}-0\right|\right) \\
& =\frac{1}{4} \sum_{j=1}^{n} w_{j}\left(\left|\mu_{i j L}\right|+\left|\mu_{i j U}\right|+\left|1-v_{i j L}\right|+\left|1-v_{i j U}\right|+\left|1-\mu_{i j U}-v_{i j U}\right|+\left|1-\mu_{i j L}-v_{i j L}\right|\right) \\
& =\frac{1}{2} \sum_{j=1}^{n} w_{j}\left(2-v_{i j L}-v_{i j U}\right)
\end{aligned}
$$

Step 4. Calculate the compromise ratio value of each alternative. 
The compromise ratio value $\xi_{i}$ represents the distances between the PIS and NIS simultaneously, and furthermore it can represent the subjective attitude of the decision maker by parameter $\varepsilon$. The compromise ratio value of each alternative is calculated as [18]:

$$
\xi_{i}=\varepsilon \frac{d_{-}\left(A^{*}\right)-d_{i}\left(A_{i}, A^{*}\right)}{d_{-}\left(A^{*}\right)-d_{+}\left(A^{*}\right)}+(1-\varepsilon) \frac{d_{i}\left(A_{i}, A^{-}\right)-d_{-}\left(A^{*}\right)}{d_{+}\left(A^{-}\right)-d_{-}\left(A^{-}\right)} \quad(i=1,2, \ldots, m)
$$

Where $d_{-}\left(A^{*}\right)=\max _{1 \leq i \leq m}\left\{d\left(A_{i}, A^{*}\right)\right\}, d_{+}\left(A^{*}\right)=\min _{1 \leq i \leq m}\left\{d\left(A_{i}, A^{*}\right)\right\}, d_{-}\left(A^{-}\right)=\min _{1 \leq i \leq m}\left\{d\left(A_{i}, A^{-}\right)\right\}$, $d_{+}\left(A^{-}\right)=\max _{1 \leq i \leq m}\left\{d\left(A_{i}, A^{-}\right)\right\}$and $\varepsilon \in[0,1]$ is the attitude factor, which represents the decision maker's subjective attitude with respect to the relative distances of alternative with PIS and NIS, and generally $\varepsilon=0.5$.

Step 5. Rank the alternatives according to the compromise ratio value $\left(\xi_{i}\right)$ in decreasing order. The larger the value of $\xi_{i}$ represents the better of the corresponding alternative $A_{i}$.

\section{Numerical Examples}

In order to illustrate the application of the proposed MADM method, two examples are given as follows:

Example 1. (This is the case of the attribute weights are complete unknown)

With the expansion of enterprise scale, enterprises need to introduce ERP management system to enhance competitiveness under the environment of economic globalization and fierce business competition,

ERP mainly lies in the integration of external resources within the enterprise, in a series of business process transformation, to improve the enterprise system, enhance the competitive ability and its content includes procurement, financial management, manufacturing, sales, research and development, human resources power module, and tts function can be further extended outward and supply chain management, customer relationship management, the combination of business intelligence. Thus ERP management system is a set of perfect function and the import process with highly complex, high risk, and import expensive suit software [8]. Based on the above characteristics, if enterprises want to conduct the software development, they must invest the massive human, physical strength, time and money etc., which are completely not in line with the economic benefit. Therefore based on the consideration of economic benefit, enterprises will take with rich experience for guiding software package suppliers, and buy the current ERP software package, to save system development costs and shorten the time to implement, fast on-line operation, to grasp business opportunities.

The expansion of enterprise scale urges the enterprise to implement ERP management. After a preliminary investigation and screening, the enterprise finally determine five candidate ERP softwares $A_{i}(i=1,2,3,4,5)$ to be choosen. Evaluated attributes ares ystem $\operatorname{cost}\left(o_{1}\right)$, functional satisfaction $\left(o_{2}\right)$, system stability $\left(o_{3}\right)$, software credibility $\left(o_{4}\right)$ and service level $\left(o_{5}\right)$. After the expert investigation and statistical analysis, the evaluation results of these 5 ERP softwares (alternatives) are obtained and expressed by IVIF numbers. Evaluated values are shown in Table 1. 
Table 1. Interval-Valued Intuitionistic Fuzzy Decision Matrix

\begin{tabular}{|c|c|c|c|c|c|}
\hline \multirow{2}{*}{$\begin{array}{c}\text { ERP } \\
\text { Software }\end{array}$} & \multicolumn{5}{|c|}{ Attributes } \\
\hline & $o_{1}$ & $O_{2}$ & $O_{3}$ & $o_{4}$ & $O_{5}$ \\
\hline$A_{1}$ & $<[0.4,0.5]$, & $<[0.5,0.6]$, & $<0.6,0.7]$ & $<[0.7,0.8]$, & $<0.7,0.8]$ \\
\hline & {$[0.4,0.5]>$} & {$[0.1,0.2]>$} & {$[0.2,0.3]>$} & {$[0.1,0.2]>$} & {$[0.0,0.2]>$} \\
\hline$A_{2}$ & $\begin{array}{l}{[0.0,0.8],} \\
{[0.1,0.2]>}\end{array}$ & $\begin{array}{l}{[0.5,0.0],} \\
{[0.3,0.4]>}\end{array}$ & $\begin{array}{l}\langle 0.4,0.5], \\
{[0.3,0.4]>}\end{array}$ & $\begin{array}{l}{[0.4,0.0],} \\
{[0.3,0.4]>}\end{array}$ & $\begin{array}{l}{[0.4,0.3],} \\
{[0.1,0.3]>}\end{array}$ \\
\hline$A_{3}$ & $\begin{array}{l}<[0.5,0.6], \\
{[0.3,0.4]>}\end{array}$ & $\begin{array}{l}<[0.5,0.7], \\
{[0.1,0.2]>}\end{array}$ & $\begin{array}{l}<[0.5,0.6], \\
{[0.3,0.4]>}\end{array}$ & $\begin{array}{l}<[0.3,0.4], \\
{[0.2,0.5]>}\end{array}$ & $\begin{array}{l}<[0.6,0.7], \\
{[0.2,0.3]>}\end{array}$ \\
\hline$A_{4}$ & $\begin{array}{l}<[0.5,0.6], \\
{[0.3,0.4]>}\end{array}$ & $\begin{array}{l}<[0.7,0.8], \\
{[0.0,0.1]>}\end{array}$ & $\begin{array}{l}<[0.4,0.5], \\
{[0.2,0.4]>}\end{array}$ & $\begin{array}{l}<[0.5,0.7], \\
{[0.1,0.2]>}\end{array}$ & $\begin{array}{l}<[0.5,0.7], \\
{[0.2,0.3]>}\end{array}$ \\
\hline$A_{5}$ & $\begin{array}{l}<[0.4,0.7], \\
{[0.2,0.3]>}\end{array}$ & $\begin{array}{l}<[0.5,0.6], \\
{[0.2,0.4]>}\end{array}$ & $\begin{array}{l}<[0.3,0.6], \\
{[0.3,0.4]>}\end{array}$ & $\begin{array}{l}<[0.6,0.8], \\
{[0.1,0.2]>}\end{array}$ & $\begin{array}{l}<[0.4,0.5], \\
{[0.2,0.3]>}\end{array}$ \\
\hline
\end{tabular}

In order to determine the best ERP management software, this paper uses the proposed method for sorting and selection of the 5 ERP management softwares.

Step 1. According to the Section 4.1, the attribute weights vector is obtained as:

$$
\boldsymbol{W}=(0.1498,0.2426,0.0803,0.2720,0.2554)^{T}
$$

Step 2.The PIS $\left(A^{*}\right)$ and NIS $\left(A^{-}\right)$are respectively given as:

$$
\begin{aligned}
& A^{*}=\left(\tilde{a}_{1}^{*}, \tilde{a}_{2}^{*}, \ldots, \tilde{a}_{5}^{*}\right)=(<[1,1],[0,0]>,<[1,1],[0,0]>, \ldots,<[1,1],[0,0]>) \\
& A^{-}=\left(\tilde{a}_{1}^{-}, \tilde{a}_{2}^{-}, \ldots, \tilde{a}_{5}^{-}\right)=(<[0,0],[1,1]>,<[0,0],[1,1]>, \ldots,<[0,0],[1,1]>)
\end{aligned}
$$

Step 3. The distance measures of each alternative from PIS and NIS are calculated as:

$$
\begin{aligned}
& d\left(A_{1}, A^{*}\right)=0.3515, d\left(A_{2}, A^{*}\right)=0.4492, d\left(A_{3}, A^{*}\right)=0.4667, \\
& d\left(A_{4}, A^{*}\right)=0.3831, d\left(A_{5}, A^{*}\right)=0.4428 \\
& d\left(A_{1}, A^{-}\right)=0.8098, d\left(A_{2}, A^{-}\right)=0.7183, d\left(A_{3}, A^{-}\right)=0.7241, \\
& d\left(A_{4}, A^{-}\right)=0.8067, d\left(A_{5}, A^{-}\right)=0.7570
\end{aligned}
$$

Step 4. Set $\varepsilon=0.5$, the compromise ratio values are calculated as:

$$
\xi_{1}=1.0000, \xi_{2}=0.0762, \xi_{3}=0.0316, \xi_{4}=0.8457, \xi_{5}=0.3157 \text {. }
$$

Therefore, the ranking order of all alternatives is $A_{1} \succ A_{4} \succ A_{5} \succ A_{2} \succ A_{3}$, and $A_{1}$ is the desirable alternative.

Example 2. (This is the case of the attribute weights are partially known.) The example is adopted from Wei [19], which considers a company's selection of outstanding management personnel.

Suppose a company wants to choose a good management personnel from 5 candidates $A_{i}(i=1,2, \ldots, 5)$. Through discussion of company managers, the final determined evaluation attributes are professional skills $\left(o_{1}\right)$, interpersonal skills $\left(o_{2}\right)$, rational skills $\left(O_{3}\right)$ and design skills $\left(o_{4}\right)$. Assuming that the evaluation information of the potential managers is given by the IVIF numbers, the corresponding IVIF decision matrix $D=\left(\tilde{a}_{i j}\right)_{m \times n}$ is shown in Table 2 . 
Table 2. Interval-Valued Intuitionistic Fuzzy Decision Matrix

\begin{tabular}{ccccc}
\hline \multirow{2}{*}{ Candidates } & \multicolumn{4}{c}{ Attributes } \\
\cline { 2 - 5 } & $o_{1}$ & $o_{2}$ & $o_{3}$ & $o_{4}$ \\
\hline \multirow{2}{*}{$A_{1}$} & $<[0.4,0.5]$, & $<[0.4,0.6]$, & $<[0.3,0.4]$, & $<[0.5,0.6]$, \\
& {$[0.3,0.4]>$} & {$[0.2,0.4]>$} & {$[0.4,0.5]>$} & {$[0.1,0.3]>$} \\
$A_{2}$ & $<[0.5,0.6]$, & $<[0.6,0.7]$, & $<[0.5,0.6]$, & $<[0.4,0.7]$, \\
& {$[0.2,0.3]>$} & {$[0.2,0.3]>$} & {$[0.3,0.4]>$} & {$[0.1,0.2]>$} \\
$A_{3}$ & $<[0.3,0.5]$, & $<[0.1,0.3]$, & $<[0.2,0.5]$, & $<[0.2,0.3]$, \\
& {$[0.3,0.4]>$} & {$[0.5,0.6]>$} & {$[0.4,0.5]>$} & {$[0.4,0.6]>$} \\
$A_{4}$ & $<[0.2,0.5]$, & $<[0.4,0.7]$, & $<[0.4,0.5]$, & $<[0.5,0.8]$, \\
& {$[0.3,0.4]>$} & {$[0.1,0.2]>$} & {$[0.3,0.5]>$} & {$[0.1,0.2]>$} \\
$A_{5}$ & $<[0.3,0.4]$, & $<[0.7,0.8]$, & $<[0.5,0.6]$, & $<[0.6,0.7]$, \\
& {$[0.1,0.3]>$} & {$[0.1,0.2]>$} & {$[0.2,0.4]>$} & {$[0.1,0.2]>$} \\
\hline
\end{tabular}

Assume the attribute weights are partially known, and the weights satisfies the set $H=\left\{0.13 \leq w_{1} \leq 0.15,0.35 \leq w_{2} \leq 0.40,0.15 \leq w_{3} \leq 0.20,0.30 \leq w_{4} \leq 0.35, w_{3} \leq w_{4}\right\}$.

Then the calculation steps of the proposed decision making method are given as follows:

Step 1. According to the Equation (19), we can establish the following programming model:

$$
\begin{aligned}
& \min E=4.9064 w_{1}+4.2861 w_{2}+4.8951 w_{3}+4.3749 w_{4} \\
& \text { s.t. }\left\{\begin{array}{l}
0.13 \leq w_{1} \leq 0.15 \\
0.35 \leq w_{2} \leq 0.40 \\
0.15 \leq w_{3} \leq 0.20 \\
0.30 \leq w_{4} \leq 0.35 \\
w_{3} \leq w_{4} \\
w_{1}+w_{2}+w_{3}+w_{4}+w_{5}=1
\end{array}\right.
\end{aligned}
$$

We use MATLAB software to solve this model, and get the optimum attribute weight vector:

$$
\boldsymbol{W}=(0.13,0.40,0.15,0.32)^{T} \text {. }
$$

Step 2. The PIS $\left(A^{*}\right)$ and NIS $\left(A^{-}\right)$are respectively given as:

$$
\begin{aligned}
& A^{*}=\left(\tilde{a}_{1}^{*}, \tilde{a}_{2}^{*}, \tilde{a}_{3}^{*}, \tilde{a}_{4}^{*}, \tilde{a}_{5}^{*}\right)=(<[1,1],[0,0]>,<[1,1],[0,0]>, \ldots,<[1,1],[0,0]>) \\
& A^{-}=\left(\tilde{a}_{1}^{-}, \tilde{a}_{2}^{-}, \tilde{a}_{3}^{-}, \tilde{a}_{4}^{-}, \tilde{a}_{5}^{-}\right)=(<[0,0],[1,1]>,<[0,0],[1,1]>, \ldots,<[0,0],[1,1]>)
\end{aligned}
$$

Step 3. The weighted Hamming distance measures of each alternative from PIS and NIS are calculated as

$$
\begin{aligned}
& d\left(A_{1}, A^{*}\right)=0.5130, d\left(A_{2}, A^{*}\right)=0.4100, d\left(A_{3}, A^{*}\right)=0.7355, \\
& d\left(A_{4}, A^{*}\right)=0.4590, d\left(A_{5}, A^{*}\right)=0.3640 \\
& d\left(A_{1}, A^{-}\right)=0.7030, d\left(A_{2}, A^{-}\right)=0.7670, d\left(A_{3}, A^{-}\right)=0.5070, \\
& d\left(A_{4}, A^{-}\right)=0.7865, d\left(A_{5}, A^{-}\right)=0.8210
\end{aligned}
$$

Step 4. Set $\varepsilon=0.5$, the compromise ratio values are calculated as:

$\xi_{1}=0.6116, \xi_{2}=0.8266, \xi_{3}=0, \xi_{4}=0.8172, \xi_{5}=1.0000$

Step 5. Based on $C_{i}$ values, the ranking of the alternatives in descending order are $A_{5} \succ A_{2} \succ A_{4} \succ A_{1} \succ A_{3}$, and $A_{5}$ is the best desirable candidate. This result is in agreement with the result in Wei [19]. However, the method presented in this paper can reflect the subjective attitude of the decision maker, and it is more consistent with the objective reality. 


\section{Conclusion}

In order to measure the fuzzy degree of IVIF sets, a new fuzzy entropy is proposed. It includes not only the absolute value of the deviation between membership and nonmembership degrees, but also the uncertainty of the intuitionistic fuzzy sets. Thus the new IVIF can better describe the degree of uncertainty and the degree of uncertainty of the IVIF sets. On this basis, the MADM problems of two kinds of situations, which the weights information of attribute are completely unknown and partially known, are sloved by developing a new MADM method on the basis of concept of compromise ratio method.

The advatage of the new proposed MADM method are descriped as follows:

(1) Reference [20] pointed out that TOPSIS method exists some shortcomings, such as reverse problem, comprehensive evaluation value reflects only the evaluation schemes in the relative proximity, which does not reflect and ideal of optimal solution is really close to the degree. To overcome these drawbacks, Li [18] proposed a compromise ratio method for fuzzy MADM problems. In this paper, we propose a compromise ratio method for MADM problems under IVIF environment based on IVIF entropy. The method can not only reflect the alternatives is close to the ideal point away from the negative ideal point, and through introducing attitude factor which is introduced as weight of the decision making strategy of "closeness to the positive ideal solution" and more in line with the actual situation.

(2) For the two cases with attribute weights information unknown and partially known, new weighting methods are put forward by using the extended entropy method and by establishing the minimum entropy optimization model to solve the optimal weights. Two examples are used and shown the proposed method is feasible and effective.

(3) The entropy measure can be applied to image processing, pattern recognition and medical diagnosis and so on, the proposed multi attribute decision making method can be applied to such as venture investment project selection, the site selection of multi attribute decision making problem, has good theoretical value and practical value.

\section{Acknowledgements}

This paper was supported by the National Natural Science Foundation of China (No. 11461029) and Natural Science Foundation of Jiangxi Province (No. 20132BAB211015 and No. 2014BAB201009). Science and Technology Research Project of Jiangxi Provincial Education Department (No. GJJ14449), and Natural Science Foundation of Jiangxi University of Science and Technology (JXUST) (No. NSFJ2014-G38).

\section{References}

[1] K. T. Atanassov, "Intuitionistic Fuzzy Sets", Fuzzy Sets and Systems, vol. 20, no. 1, (1986), pp. 87-96.

[2] K. T. Atanassov and G. Gargov, "Interval-valued intuitionistic fuzzy sets", Fuzzy Sets and Systems, vol. 31, no. 3, (1989), pp. 343-349.

[3] T. Y. Chen, "The inclusion-based TOPSIS method with interval-valued intuitionistic fuzzy sets for multiple criteria group decision making", Applied Soft Computing, vol. 26, (2015), pp. 57-73.

[4] S. P. Wan and J. Y. Dong, "Interval-valued intuitionistic fuzzy mathematical programming method for hybrid multi-criteria group decision making with interval-valued intuitionistic fuzzy truth degrees", Information Fusion, vol. 26, (2015), pp. 49-65.

[5] S. M. Chen, L. W. Lee, H. C. Liu and S. W. Yang, "Multiattribute decision making based on intervalvalued intuitionistic fuzzy values", Expert Systems with Applications, vol. 39, no. 12, (2012), pp. 10343-10351.

[6] L. A. Zadeh, "Probability measures of fuzzy events", Journal of Mathematical Analysis and Applications, vol. 23, no. 2, (1968), pp. 421-427.

[7] A. D. Luca and S. Termini, "A definition of non-probabilistic entropy in the setting of fuzzy set theory", Information Control, vol. 20, no. 4, (1972), pp. 301-312. 
[8] X. W. Qi, C. Y. Liang, A. Q. Zhang and Y. Ding, "Approach to interval-valued intuitionistic fuzzy multiple attributes group decision making based on maximum entropy", Systems Engineering-Theory \& Practice, vol. 31, no. 10, (2011), pp. 1940-1948.

[9] Q. Liu, L. P. Xu, Y. D. Ma, Z. Su and Y. Wang, "Automated image segmentation using the ULPCNN model with ultra-fuzzy entropy", Journal of Xidian University, vol. 37, no. 5, (2010), pp. 817-824.

[10] X. Y. Zou and M. Lei, "Pattern recognition of surface electromyography signal based on multiscale fuzzy entropy", Journal of Biomedical Engineering, vol. 2, no. 6, (2012), pp. 1184-1188.

[11] [11] J. Wang and P.Wang, "Intuitionistic linguistic fuzzy multi-criteria decision-making method based on intuitionistic fuzzy entropy", Control and Decision, vol. 27, (2012), pp.1694-1698.

[12] Z. S. Xu, "Intuitionistic fuzzy hierarchical clustering algorithms", Journal of System Engineering and Electronics, vol. 20, no. 1, (2009), pp. 90-97.

[13] C. P. Wei, P. Wang and Y. Z. Zhang, "Entropy, similarity measure of interval-valued intuitionistic fuzzy sets and their applications", Information Sciences, vol. 181, no. 19, (2011), pp. 4273-4286.

[14] I. K. Vlachos and G. D. Sergiadis, "Subsethood, entropy, and cardinality for interval-valued fuzzy setsan algebraic derivation", Fuzzy Sets and Systems, vol. 158, no. 12, (2007), pp. 1384-1396.

[15] J. Ye, "Multicriteria fuzzy decision-making method using entropy weights-based correlation coefficients of interval-valued intuitionistic fuzzy sets", Applied Mathematical Modelling, vol. 34, no. 12, (2010), pp. 3864-3870.

[16] F. F. Jin, L. D. Pei, H. Y. Chen and L. G. Zhou, "Interval-valued intuitionistic fuzzy continuous weighted entropy and its application to multi-criteria fuzzy group decision making", Knowledge-Based Systems, vol. 59, (2014), pp. 132-141.

[17] M. F. Liu and H. P. Ren, "A new intuitionistic fuzzy entropy and application in multi-attribute decision making", Information, vol. 5, no. 4, (2014), pp. 587-601.

[18] D. F. Li, "Compromise ratio method for fuzzy multi-attribute group decision making", Applied Soft Computing, vol. 7, no. 3, (2007), pp. 807-817.

[19] G. W. Wei, "Theory and Methods of Multiple Attribute Decision Making Based on Fuzzy Information", Beijing, China Economic Publishing House, (2010).

[20] Y. R. Wang, L. F. Ren, L. W. Chen and Z. Q. Sun, "A novel improved TOPSIS method and its application in medical science", Journal of Central South University (Medical Sciences), vol. 38, no. 2, (2013), pp. 196-201.
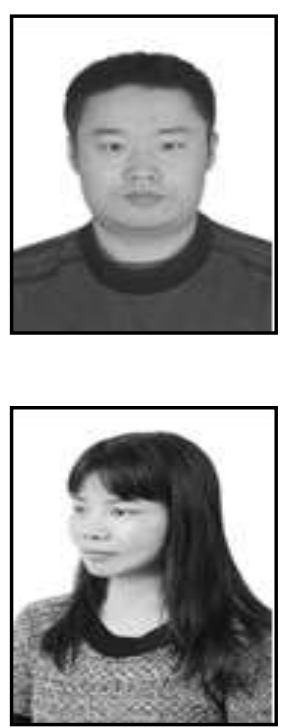

\section{Authors}

Haiping Ren, he is an associate professor of Jiangxi University of Science and Technology. He obtained his bachelor degree of applied mathematics in 2003 from Changsha University Science and Technology, master degree of probability and statistics in 2005 from Central South University, and Ph.D. of management science and engineering in 2015 from Jiangxi University of Finance and Economics. His major researches are Bayes statistics, fuzzy making and information fusion.

Wanzhen Liu, she is a lecture of Changsha Vocational \& Technical College. She obtained her bachelor degree of applied mathematics in 1996 from Hunan Normal University. She has published more than 10 articles in fuzzy attribute decision making and Bayesian statistics. Now, her major researches are mainly Bayes statistics, fuzzy making and information fusion. 
International Journal of Hybrid Information Technology

Vol. 10, No.1 (2017) 